\title{
Broadcasting Monitoring of SSR Transponders in Real Space
}

\section{Vít Vojtěch ${ }^{1 *}$, Stanislav Pleninger ${ }^{1}$}

\author{
${ }^{1}$ Department of Air Transport, Faculty of Transportation Sciences, Czech Technical University in Prague, Prague, \\ Czech Republic \\ *Corresponding author: Czech Technical University in Prague, Faculty of Transportation Sciences, Department of Air \\ Transport, Horská 3, 12803 Prague, Czech Republic, Email: vitvojtech@gmail.com
}

\begin{abstract}
The article presents a method that allows achieved the greater completeness of the record during the reconstruction of emitted SSR transponder messages captured by terrestrial receivers. The method is based on a fusion of records from several ground-based receivers. The second part of the article presents and evaluates the results achieved using this method on the network of the low-cost ADS-B receivers in the Department of Air Transport, Czech Technical University in Prague Faculty of Transportation Sciences. Some inevitable phenomena are demonstrated that this measurement method brings.
\end{abstract}

\section{Keywords}

SSR Mode S; SSR transponder; 1090ES; ADS-B receiver; Secondary Surveillance Radar; Method for message reconstruction

\section{Introduction}

This article describes method created for reconstruction of Mode $\mathrm{S}$ transponders broadcasting. The method is based on fusion of messages received by low cost ADS-B receivers which are used for science purposes by Faculty of Transportation Science. The part of article deals with evaluation of method dependence on several factors.

Data from real traffic were used for application of method. For this reason, receiving ability of each receiver which was used for data collection can be evaluated by application of the method based on several parameters.

One of the ADS-B broadcast technology is 1090ES (Extended Squitter). Mentioned technology uses the same broadcast frequency as SSR Mode S Transponders. This fact is the reason why it is possible to receive SSR Mode S transponders broadcasting by ADS-B receivers.

\section{Method for reconstruction of Mode S transponders broadcasting}

The purpose of created method is to provide the most accurate reconstruction of the SSR transponders broadcasting based on data from low cost ADS-B receivers. Data from four ADS-B receivers which are placed in different areas in Prague were used for method realisation. The algorithm for reconstruction is described in the following text and the algorithm steps are summarized in Table 1 bellow.

First off all it is necessary to decode DF (Downlink format) of each message. Decoding of Downlink format as a first step 
is important because transponder ICAO address is decoded based on Downlink format. Next step is to decode time of the message receive and position of the aircraft including altitude. Information about aircraft's position isn't contained in each message. In created method, position is obtained from DF 17 messages with Type code value between 9 and 18. DF 17 message format is designed for use by ADS-B messages. Position messages are key element for message reconstruction.

For required accuracy of outputs it is necessary to make transformation of aircraft and receiver's coordinates from Geographic coordinate system to Cartesian coordinate system.

For unification of all messages received by several receivers it is necessary to recalculate receive time of messages on individual receiver to one point. In the proposed method, receive time of each message were recalculated to aircraft. At this step of algorithm, position messages are already decoded.

From knowledge of aircraft position and each receiver position it is possible to calculate oblique distance between aircraft and receiver at the receive time of message by equation 1. With decoded received time from previous step of method and known duration of electromagnetic wave emission (from equation 2), it is possible to compute emitting time of the message by equation 3 .

$$
D=\sqrt{\left(X_{A}-X_{R}\right)^{2}+\left(Y_{A}-Y_{R}\right)^{2}+\left(Z_{A}-Z_{R}\right)^{2}}
$$

$D$ is oblique distance between Aircraft and Receiver. $X$, $Y, Z$ are Aircraft (A) and Receiver (R) coordinates.

$$
T_{E M R}=\frac{D}{C}
$$

$T_{E M R}$ is duration of wave emission. $C$ is speed of electromagnetic radiation in free space.

$$
T_{A}=T_{R}-T_{E M R}
$$

$T_{A}$ is emitting time of message by aircraft transponder. $T_{R}$ is time of receive which is know from previous step of the method.

Mentioned method of time computing can be applied for position messages but information about aircraft position isn't contained in each message. Emitting time of all messages is necessary for next steps of algorithm.

To compute emitting time of messages without position, time difference between receive time of message with position and of message without position is used. Time difference is subsequently added or deducted to already known emitting time of message with position. After this step, emitting time for all messages is known.

In real environment, it is common that one emitted message is received by several receivers. For reconstruction of emitted messages by more than one receiver, it is necessary to identify duplicity messages and don't count them in following analysis. Identification of duplicity messages works on the
Table 1. Algorithm for reconstruction of Mode S transponder broadcasting in steps, source: author

\begin{tabular}{ll}
\hline Step & Operation \\
\hline 1 & Downlink format and ICAO address decoding \\
2 & Receive time decoding \\
3 & Aircraft position and altitude decoding \\
4 & Aircraft and receivers coordinate transformation \\
5 & Calculation of emitting time for position messages \\
6 & Calculation of emitting time for non-position messages \\
7 & Identification of duplicity messages \\
8 & Identification of bounced messages \\
\hline
\end{tabular}

Table 2. DF 17 message broadcasting rate, source: Minimum Operational Performance Standards for $1090 \mathrm{MHz}$ [1]

\begin{tabular}{clcc}
\hline Type Code & Type of Message & Message broadcasting rate & Number of emitted messages per one minute \\
\hline $1-4$ & Aircraft Identification Message & 5 second & 12 \\
$9-18$ & Aircraft Position Message & 0,5 second & 120 \\
19 & Aircraft Velocity Message & 0,5 second & 120 \\
\hline
\end{tabular}

presumption that aircraft transponder can't emit two messages in one moment. Based on this hypothesis, messages are duplicity if difference between emitting time of two messages is shorter than length of compared messages.

Another type of messages which can't be counted to analysis are bounced messages. Bounced message is a message that was received more than once at one receiver. Mentioned phenomena is caused by message bounce from obstacle. For that reason bounced messages are more common in the case that antenna of receiver is placed close to high building or another obstacle.

\section{Method succes rate evaluation}

The success of the created method for reconstruction of Mode $\mathrm{S}$ transponders broadcasting is evaluated by comparing successfully reconstructed messages after fusion of reconstructed messages from all used receivers without duplicity and bounced messages and known number of DF 17 messages which are transmitted by on-board transponders using 1090ES technology. The number of DF17 messages is stated in document Minimum Operational Performance Standards for $1090 \mathrm{MHz}$ [1] and is shown in Table 2.

\subsection{Method success rate evaluation for low density traffic}

For method evaluation in low density traffic were used data received by ADS-B receivers from $3 \mathrm{am}$. Seven flights in different distances from receiver system were chosen from data record. Such choice was made for purpose to evaluate success rate of reconstruction depending on distance of aircraft from receivers. Table 3 shows percentage success of reconstruction for each Type Code in one minute period. $100 \%$ value of reconstructed messages with Type code between 9-18 on flight $45201 \mathrm{C}$ indicates that 120 out of 120 DF 17 messages with Type Code 9-18 per one minute were successfully reconstructed. Dependence of successful reconstruction on distance 
Table 3. Success rate of DF 17 messages reconstruction for low density traffic, source: author

$$
\begin{array}{llllllll} 
& \multicolumn{7}{c}{\text { Success rate of DF 17 messages reconstruction }} \\
\text { Type Code } & 45201 \mathrm{C} & 48455 \mathrm{~F} & 484371 & 44 \mathrm{D} 074 & 3 \mathrm{C} 4588 & 47 \mathrm{~A} 41 \mathrm{~B} & 3 \mathrm{C} 4981 \\
1-4 & 100,0 \% & 83,3 \% & 41,7 \% & 83,3 \% & 33,3 \% & 33,3 \% & 83,3 \% \\
9-18 & 100,0 \% & 89,2 \% & 33,3 \% & 95,8 \% & 30,0 \% & 27,5 \% & 83,3 \% \\
19 & 96,7 \% & 86,7 \% & 37,5 \% & 92,5 \% & 29,2 \% & 16,7 \% & 80,0 \% \\
\text { Average } & 98,9 \% & 86,4 \% & 37,5 \% & 90,6 \% & 30,8 \% & 28,3 \% & 82,2 \%
\end{array}
$$

of aircraft from receivers in low density traffic is represent by trend curve on Figure 1.

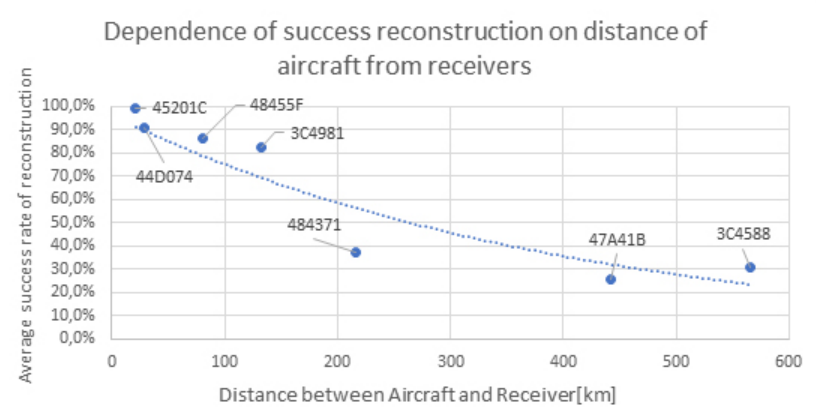

Figure 1. Dependence of reconstruction on distance of aircraft from receivers in low density traffic, source: author

Distance between aircraft and receiver at the moment of first DF17 position message received from the aircraft is shown on the axis $\mathrm{X}$.

Four ADS-B receivers were used for the reconstruction. First receiver named Horská has with only 26,9\% of messages received on average the worst success rate. The success rate is calculated as ratio between number of messages received by receiver Horská and number of all reconstructed messages after fusion of data from four used receivers. Second receiver Pankrác has success rate of receiving 32,8 \%. Third receiver Letňany has success rate of receiving 59,9 \%. Receiver Strahov has the best success rate of all receivers used with 71,0\% of successfully received messages.

Receiver Letňany has the lowest bounce rate with $0,7 \%$ of bounced messages from all messages received by mentioned receiver. Receiver Strahov has the highest bounce rate (the worst result) with $4,2 \%$ bounced messages from all messages received by receiver Strahov.

\subsection{Method succes rate evaluation for high density traffic}

To evaluate the method in high density traffic, were used data received by ADS-B receivers from 6:40 am. Seven flights in different distances from receiver system were chosen for high density traffic. The percentage of success of reconstruction for each Type Code in one-minute period is shown in Table 4. Dependence of successful reconstruction on distance of aircraft from receivers in high density traffic is presented in Figure 2.
Table 4. Success rate of DF 17 messages reconstruction for high density traffic, source: author

\begin{tabular}{llllllll}
\hline \multirow{2}{*}{ Type Code } & \multicolumn{7}{c}{ Success rate of DF 17 messages reconstruction } \\
& 02A1AF & 45AA84 & 49D095 & 42491F & 4BCDD2 & 7380AA & 3 366488 \\
\hline $1-4$ & $58,3 \%$ & $50,0 \%$ & $91,7 \%$ & $83,3 \%$ & $75,0 \%$ & $91,7 \%$ & $83,3 \%$ \\
$9-18$ & $73,3 \%$ & $55,0 \%$ & $93,3 \%$ & $78,3 \%$ & $61,7 \%$ & $90,0 \%$ & $80,8 \%$ \\
19 & $60,0 \%$ & $59,2 \%$ & $92,5 \%$ & $77,5 \%$ & $56,7 \%$ & $93,3 \%$ & $80,8 \%$ \\
Average & $63,9 \%$ & $54,7 \%$ & $92,5 \%$ & $79,7 \%$ & $64,4 \%$ & $91,7 \%$ & $81,7 \%$ \\
\hline
\end{tabular}

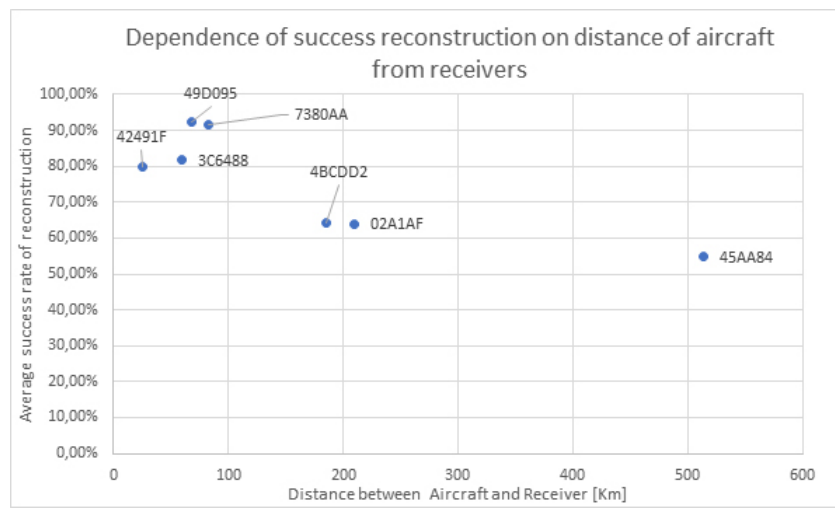

Figure 2. Dependence of reconstruction on distance of aircraft from receivers in high density traffic, source: author

Distance between aircraft and receiver in the moment of first DF17 position message received from the aircraft is shown on the axis $\mathrm{X}$.

The same four ADS-B receivers were used for reconstruction in both high and low-density traffic. Again, receiver Horská scores the worst among other receivers with the average success rate of receiving of $7,9 \%$. Success rate of receiving of receiver Pankrác is 35,9\%. Receiver Letňany success rate of receiving is $60,7 \%$. Receiver Strahov with $71,4 \%$ of success rate of receiving has the best success rate from all used receivers.

Receiver Letňany with $0,4 \%$ of bounced messages from all received messages by mentioned receiver has the lowest bounce rate. Strahov receiver with $4,7 \%$ bounced messages from all received messages by receiver Strahov has the highest bounce rate (the worst result).

\section{Discussion}

The created method is dependent on receiving ability of each ADS-B receiver in system. Density of traffic is one of the factors which influence receiving ability of receiver. Receivers are strained by big amount of incoming messages in heavy traffic which affect success receive of messages. This fact was proved by average success rate of reconstruction in Figure 1 and Figure 2. Average success rate of reconstruction in low density traffic for distance up to $100 \mathrm{~km}$ from receiver is more than $90 \%$ whereas average success rate of reconstruction in high density traffic for same distances is only $86,4 \%$.

Dependence of successful reconstruction on distance of aircraft from receiver in low density traffic is represented by trend curve shown in Figure 1. As shown in Figure 2 the dependence in high density traffic isn't so definite as in 
low density traffic. The volume of incoming messages is the reason why average success rate of reconstructed messages in high traffic isn't as regular as reconstruction in low traffic. Higher volume of messages affects negatively the success of received messages - the number of reconstructed message is lower. Receiver Horská has the worst success rate of receiving in both low density and in high density traffic. Receiver Horská has success rate of receiving on average $26,9 \%$ for low density traffic and only $7,9 \%$ for high density traffic. The main reason for low success rate is the fact that some of the chosen aircrafts were out of receiver range - this fact is caused by the lowest range of receiver Horská out of all receivers. Low range of a receiver can have positive influence on message reconstruction. Receivers with low range aren't strained by such a large amount of incoming messages as high range receivers which receive messages from aircrafts from bigger area. Receiver Strahov has the best success rate of receiving in low density and in high density traffic. Receiver Strahov has average success rate of receiving $71,0 \%$ for low density traffic and $71,4 \%$ for high density traffic.

Receiver Letňany has the lowest bounce rate with $0,7 \%$ of bounced messages from all messages received on this receiver in low density traffic and only $0,4 \%$ in high density traffic. The reason lies on the position of the receiver which antenna is placed in suitable area without obstacles. Strahov receiver has the highest bounce rate from all used receivers. There are $4,2 \%$ bounced messages from all received messages in low density traffic and 4,7\% in high density traffic. More frequent bounces of messages in this receiver are caused by high building surrounding receiver's antenna.

\section{Conclusion}

The main goal was to create method for reconstruction of Mode $\mathrm{S}$ transponders broadcasting. Article describes the main factors that influence success of reconstruction. Data for method realisation were obtained from Faculty of Transportation Science ADS-B receivers. Receiving success rate of used receivers is one output of the method which can be used for optimization of ADS-B receiver system or serve as a source for further students' projects.

\section{References}

[1] Inc. RTCA. Minimum operational performance standards for $1090 \mathrm{mhz}$ extended squitter automatic dependent surveillance - broadcast (ads-b) and traffic information services - broadcast (tis-b), 2009. URL http://www.anteni.net/adsb/Doc/ 1090-WP30-18-DRAFT_DO-260B-V42.pdf.

[Online]. 